\title{
A Combined Proteomics and Metabolomics Profiling to Investigate the Genetic Heterogeneity of Autistic Children
}

\section{Liming Shen}

Shenzhen University

Huajie Zhang

Shenzhen University

Jing Lin

Shenzhen University

Yan Gao

Maternal and Child Health Hospital of Baoan

Xukun Liu

Shenzhen University

\section{Margy Chen}

Emory University

\section{Naseer Ullah Khan}

Shenzhen University

\section{Xiaoxiao Tang}

Shenzhen University

\section{Qi Hong}

Maternal and Child Health Hospital of Baoan

\section{Chengyun Feng}

Maternal and Child Health Hospital of Baoan

\section{Yuxi Zhao}

Shenzhen University

xueshan cao ( $\square$ cxsh1021@szu.edu.cn)

Shenzhen University https://orcid.org/0000-0002-0905-8239

\section{Research}

Keywords: Autism spectrum disorder, metabolomics, heterogeneity, plasma, peripheral blood mononuclear cells, proteomics, biomarker

Posted Date: October 12th, 2021 
DOl: https://doi.org/10.21203/rs.3.rs-944640/v1

License: (c) (1) This work is licensed under a Creative Commons Attribution 4.0 International License. Read Full License

Version of Record: A version of this preprint was published at Molecular Neurobiology on March 28th, 2022. See the published version at https://doi.org/10.1007/s12035-022-02801-x. 


\section{Abstract}

Background: Autism spectrum disorder (ASD) has become one of the most common neurological developmental disorders in children. However, the study of ASD diagnostic markers faces significant challenges due to the existence of heterogeneity.

Methods: In this study, genetic testing was performed on children who were clinically diagnosed with ASD. Children with ASD susceptibility genes and healthy controls were studied. The proteomics of plasma and peripheral blood mononuclear cells (PBMCs) as well as plasma metabolomics were carried out.

Results: The results showed that although there was genetic heterogeneity in children with ASD, the differentially expressed proteins (DEPs) in plasma, PBMCs, and differential metabolites in plasma could still effectively distinguish autistic children from controls. The mechanism associated with them focus on several common and previously reported mechanisms of ASD.

Limitations: The number of samples carrying risk genes in omics research is limited. In further research, a large sample size is required. A group of children who have been diagnosed with ASD but have not been detected to carry risk genes should also be included.

Conclusion: The biomarkers for ASD diagnosis could be found by taking DEPs and differential metabolites into consideration. Integrating omics data, glycerophospholipid metabolism and N-glycan biosynthesis might play a critical role in the pathogenesis of ASD.

\section{Introduction}

Autism spectrum disorder (ASD) is a group of developmental neurological disorders characterized by early onset of abnormal social communication and restricted repetitive behaviors and interests. In recent years, its incidence has gradually increased. ASD is about four times more common among boys than girls ${ }^{1}$. Although its pathogenesis has not been clarified, studies have shown that it may be a multifactorial disorder. Twin studies suggest genes play a key role in the pathogenesis of ASD $^{2}$. It is estimated that ASD may involve thousands of genes ${ }^{1}$. However, ASD is highly heterogeneous, with each gene individually accounting for less than $1 \%$ of cases $^{3}$. ASD may also be caused by the environmental factors. Therefore, it may be caused by interaction between genes and environmental factors ${ }^{1}$.

Currently, there is no specific treatment for ASD. Studies show that early intervention can significantly improve ASD symptoms of diagnosed children and help them transition into society ${ }^{4,5}$. Early diagnosis is crucial for early detection and early intervention for autistic children. However, at present, there is a lack of effective biomarkers for clinical diagnosis ${ }^{1,6-8}$. The diagnosis of ASD is based on the fifth edition of the Diagnostic and Statistical Manual of Mental Disorders (DSM-V) ${ }^{9}$. This standard brings a certain level of subjectivity into diagnosis, and is not entirely conducive to early detection of the disease. 
The plasma contains abundant substances that are convenient for sampling. Thus, it becomes a suitable candidate when screening diseases. In the search for reliable biomarkers for ASD, some studies on blood protein- or metabolites-based biomarkers have been carried out ${ }^{1,6-8}$. Some other studies suggest that peripheral blood cells such as peripheral blood mononuclear cells (PBMCs) ${ }^{10,11}$ and lymphoblastoid cell lines (LCLs) ${ }^{12}$ may become useful tools to investigate systemic neurochemical changes in ASD. However, due to the existence of heterogeneity, finding peripheral blood or cell markers for the diagnosis of ASD can be challenging. Heterogeneity in etiology, phenotype, and outcome are hallmarks of ASD $^{13}$. This may be due to the fact that ASD is a multifactorial disease. Heterogeneity complicates the quest for personalized medicine in ASD. Combining data from autistic children with different etiologies may lead to confusing results. Factors such as genetic variation, comorbidities, and gender contribute to the heterogeneity of ASD. Among them, genetic variation is the main contributor.

To explore the influence of gene heterogeneity on the search for diagnostic markers of ASD, we performed genetic testing on 122 children clinically diagnosed with ASD in the present study. Five children of the sample were found to be carrying ASD susceptibility genes. The plasma and PBMCs of these children and their gender- and age-matched healthy controls were collected, plasma and PBMCs proteomics and plasma metabolomics analysis were carried out. Based on protein and metabolite profiles collected, the diagnostic markers and the pathogenesis of ASD were studied (Fig. 1).

\section{Materials And Methods}

\section{A new generation of sequencing technology for targeted sequencing of autistic candidate genes}

An overview of the workflow used in this study is shown in Fig. 1. One hundred and twenty-two autistic children (2-6 years old) were recruited from the Maternal and Child Health Hospital of Baoan in Shenzhen, China. The diagnosis of ASD was based on the criteria of autistic disorders as defined in the DSM-囚 by a child neuropsychiatrist.

EDTA (Ethylenediaminetetraacetic acid) anticoagulation tubes were used to collect $4 \mathrm{~mL}$ of peripheral fasting blood from children in the morning. Genomic DNA was extracted from fresh blood samples using the phenol-chloroform extraction method. DNA samples were stored in anhydrous ethanol dissolved in TE buffer and diluted to $50 \mathrm{ng} / \mu \mathrm{L}$ for subsequent experiments. The molecular inversion probe (MIP) was used to design probes for autism risk genes as previously described ${ }^{14}$. The target genes were captured, digested, and amplified by PCR (Polymerase chain reaction). After enrichment and purification of the product, high-throughput sequencing was carried out on a hiseq 3000 platform (Illumina, USA). The original sequencing data were then processed by quality control, comparison, and filtration. The mutation sites were annotated and analyzed for function.

\section{Proteomics analysis of plasma and peripheral blood mononuclear cells}




\section{Protein sample preparation}

Subsequently, we performed plasma and PBMCs proteomics studies on the children with de novo mutations, along with gender- and age-matched healthy controls (Table S1). $4 \mathrm{~mL}$ of fasting blood samples were collected in EDTA-coated plastic tubes. Plasma was separated by centrifugation at $800 \times \mathrm{g}$ for $10 \mathrm{~min}$ at $4{ }^{\circ} \mathrm{C}$, and the supernatant was collected and centrifuged at $10,000 \times \mathrm{g}$ for $30 \mathrm{~min}$ at $4{ }^{\circ} \mathrm{C}$, and then divided into aliquots and stored immediately afterwards at $-80^{\circ} \mathrm{C}$. PBMCs were separated by density gradient centrifugation using Ficoll-Hypaque (Sigma-Aldrich, St. Louis, MO, USA) ${ }^{11}$.

For proteomics analysis, the plasma samples were processed using Multiple Affinity Removal Column (Hu6; MARC; 4.6×50 mm, Agilent, Palo Alto, CA, USA) as previously described ${ }^{15}$. PBMCs were lysed in RIPA lysis buffer (Beyotime, Shanghai, China), sonicated 10 times for $5 \mathrm{~s}$ with 10 s pause interval in an icewater bath, and then centrifuged at $10,000 \times \mathrm{g}$ at $4^{\circ} \mathrm{C}$ for $60 \mathrm{~min}^{11}$. The supernatant was taken away and stored at $-80^{\circ} \mathrm{C}$ until use. The protein concentrations were determined and optimized by Bradford assay.

\section{iTRAQ labeling and high-pH RPLC fractionation}

The iTRAQ analysis was performed according to the previous study ${ }^{11,15}$. The protein extraction $(100 \mu \mathrm{g})$ of plasma or PBMCs from each child with ASD and controls was reduced, alkylated, and digested with trypsin (Promega, Madison, WI, USA) at a ratio of $1: 30$ at $37^{\circ} \mathrm{C}$ overnight, and then labeled with iTRAQ reagents (AB Sciex, Foster City, CA, USA). The information of iTRAQ tags for plasma and cells is shown in Table S2. iTRAQ analysis included set A and set B. After labeling, these were incubated at room temperature for $2 \mathrm{~h}$, and then mixed and lyophilized. The dried samples were reconstituted in $100 \mu \mathrm{L}$ double-distilled water $\left(\mathrm{ddH}_{2} \mathrm{O}\right)$ and injected into the Agilent HPLC (high-performance liquid chromatography; Agilent Technologies, USA) with a high pH RP (reverse phase) column (Durashell, C18, $250 \mathrm{~mm} \times 4.6 \mathrm{~mm}, 5 \mu \mathrm{m}$; Bonna-Agela Technologies Inc., USA). Peptides were eluted and combined into 16 groups and lyophilized.

\section{NanoLC-mass spectrometry (MS)/MS analysis}

An Ultra 2D Plus nanoflow HPLC (Eksigent Inc., Dublin, CA, USA) coupled with Triple TOF 5600 system (AB Sciex) was used for the analytical separation of peptides ${ }^{11,15}$. Microfluidic traps and nanofluidic columns packed with ChromXP C18 $(3 \mu \mathrm{m}, 350 \mu \mathrm{m} \times 0.5 \mathrm{~mm}$, Eksigent) were utilized for online trapping and desalting, while nanofluidic columns packed with ChromXP C18 $(3 \mu \mathrm{m}, 75 \mu \mathrm{m} \times 150 \mathrm{~mm}$, Eksigent) were employed in analytical separation. The MS data were acquired in the positive ion mode, with a selected mass range of $350-1500 \mathrm{~m} / \mathrm{z}$. Peptides with +2 to +5 charge states were selected for MS/MS. MS/MS spectra were acquired in the mass range of $100-1500 \mathrm{~m} / \mathrm{z}$. Smart information-dependent acquisition (IDA) was activated with automatic collision energy and automatic MS/MS accumulation.

\section{Database search, ITRAQ quantification and bioinformatics analysis}


Protein identification and quantification were performed using ProteinPilot v5.0 (AB Sciex). The quantitative data were loaded to OMICSBEAN ${ }^{16}$ website. Data were normalized and t-test was performed. The cutoff value for up-regulation was 1.2-fold change, for down-regulation was 0.83 fold change, and FDR (false discovery rate) $p$-value $<0.05$ was established for significantly differentially expressed proteins (DEPs) between autistic children and controls ${ }^{17}$. Principal component analysis (PCA) was performed by using MetaboAnalyst 4.0 ${ }^{17,18}$. Partial Least Squares Discriminant Analysis (PLS-DA) were performed using SIMCA-P 14.1 software package (V14.1, Sartorius Stedim Data Analytics AB, Umea, Sweden). The model was validated using a 7 -fold cross validation method and tested with 200 random permutations. The DEPs were loaded to String ${ }^{19}$ database for biological process (BP), cellular component (CC), KEGG and REACTOME pathways analysis. Protein-protein interaction (PPI) networks were analyzed using Cytoscape 3.8.2 and STRING.

\section{Metabolomics analysis of plasma}

\section{Plasma pretreatment and mass spectrometry analysis}

Internal standard (L-2-chlorophenylalanine, $0.3 \mathrm{mg} / \mathrm{mL}$, methanol) was pre-mixed with enough methanolacetonitrile $(2: 1, v / v)$ as the metabolite extract, which was pre-cooled at $-20^{\circ} \mathrm{C}$. For each sample, $100 \mu \mathrm{L}$ plasma was mixed with $300 \mu \mathrm{L}$ methanol-acetonitrile $(2: 1, \mathrm{v} / \mathrm{v})$, sonicated in an ice water bath for $10 \mathrm{~min}$, stood at $-20^{\circ} \mathrm{C}$ for $30 \mathrm{~min}$, and then centrifugated at $4{ }^{\circ} \mathrm{C}$ at $13,000 \times \mathrm{g}$ for $15 \mathrm{~min}$. Additionally, $100 \mu \mathrm{L}$ supernatant was filled into a glass injection flask, and mixed with $10 \mu \mathrm{L}$ supernatant from each sample as quality control (QC) sample.

A Waters Acquity UPLC system coupled with a Q-TOF Synapt G2 high-definition mass spectrometer (Waters, USA) was used for metabolomics analysis. Chromatographic separation was performed on an ACQUITY UPLC BEH C18 column $(2.1 \times 100 \mathrm{~mm}, 1.7 \mu \mathrm{m})$ at $45^{\circ} \mathrm{C}$. Mobile phase $A$ was an aqueous solution containing $0.1 \%$ formic acid, and mobile phase $B$ was an acetonitrile solution containing $0.1 \%$ formic acid. The flow rate of mobile phase $A$ and $B$ was $0.4 \mathrm{~mL} / \mathrm{min}$. The gradient elution is shown in Table S3. The injection volume was $2 \mu \mathrm{L}$. The MS analysis was carried out by the ESI ion source, and the positive and negative ion scanning modes were used to collect the signal respectively. The parameters of MS analysis are shown in Table S4. Processing of the results of metabolomics mass spectrometry

The raw spectrum was processed by Progenesis QI 2.0 software (Nonlinear Dynamics, Newcastle, UK) for peak selection, comparison, standardization, and identification. $\mathrm{R}$ package metaX was used to perform further statistical analysis on the normalized peak intensity. We also used public spectral library MassBank, HMDB, LipidBlast, METLIN for secondary identification. After characterization, duplicate qualitative results are eliminated based on the score.

\section{Metabolomics data analysis}

The profile of metabolomics data was analyzed using multivariate statistical methods. PCA and PLS-DA analysis were carried out. VIP (variable importance in projection) score of PLS-DA was obtained. The 
metabolites with VIP scores $\geq 1$, fold change $\geq 1.2$ or $\leq 0.83$ (ASD versus control) as the threshold, and $p$ $<0.05$ were identified as differential metabolites. Pathway analysis were performed using MetaboAnalyst 4.0 .

\section{Integrated analysis of DEPs and differential metabolites identified in this study}

The DEPs identified by proteomics analysis between the cases and controls, including the DEPs of plasma,PBMCs, and the differential metabolites between the two groups identified by plasma metabolomics were loaded to MetaScape v3.1.3 in Cytoscape 3.4.0 for integrated analysis ${ }^{20}$.

\section{Results}

\section{The results of gene testing}

By genetic testing, among the 122 autistic patients, 5 children were found to be associated with de novo mutations in autism risk genes, including histone-lysine N-methyltransferase ASH1L (ASH1L), ATPdependent RNA helicase DDX3X (DDX3X), N-alpha-acetyltransferase 15, NatA auxiliary subunit (NAA15), GRB10-interacting GYF protein 2 (GIGYF2), and sodium channel protein type 2 subunit alpha (SCN2A), respectively. Another child was found to be associated with the mutations of the MECP2 (methyl-CpGbinding protein 2) gene. Since MECP2 is associated with Rett syndrome, which has been removed from ASD based on DSM- $\$ diagnostic criteria, so we have only further studied the other five children.

\section{The results of plasma iTRAQ quantitative proteomics analysis}

By proteomics analysis, 300 and 350 plasma proteins were identified in the set A experiment and set B experiment, respectively. Among these, 280 proteins were identified as common. The cluster and PCA analysis showed that the expression pattern of plasma proteins was heterogeneous. The characteristics of total plasma protein failed to distinguish ASD individuals from healthy controls (Fig. 2A and B). Out of the total proteins, 13 proteins were identified as DEPs between children with ASD and healthy controls (Fig. 2C, Table S5). Among them, 5 proteins were up-regulated and 8 proteins were down-regulated. The cluster analysis showed that these DEPs could distinguish the ASD group from the control group. However, the five controls were not completely clustered together, and the expression characteristics of the controls with similar age were more similar (Fig. 2D).

\section{The results of PBMCs quantitative proteomics analysis}

By proteomics analysis, 2800 proteins were identified as common in the set A experiment and set B experiment. Cluster and PCA analysis showed that the expression characteristics of total proteins failed to distinguish the ASD group from the control group (Fig. 3A and B). By comparative analysis, 111 proteins were identified as DEPs between children with ASD and controls (Fig. 3C, Table S6). Compared 
with the controls, 88 proteins were up-regulated and 22 proteins were down-regulated in the ASD individuals. Cluster analysis showed that the ASD group and control group could be well distinguished by these DEPs (Fig. 3D). Of note, among the DEPs identified in PBMCs, the genes encoding 4 DEPs (SLC9A9, CSDE1, CCT4, and DDX3X) are included in the SFARI (Simons Foundation Autism Research Initiative) gene database (https://gene.sfari.org/). Interestingly, the DDX3X gene has been detected in gene testing as described above.

\section{Bioinformatics analysis of DEPs in plasma and PBMCs}

For the DEPs of plasma, the BP and KEGG pathway are shown in the Table S7 and S8. These DEPs were involved in complement activation, immune response, and complement and coagulation cascades (Fig. 4A and B). Interestingly, some complement proteins were related to synapse punting (C1QA and C1QB) and neuronal development and plasticity (C1QA, C1QB, C1S, C2, and COLEC10).

For the DEPs of PBMCs, the BP, KEGG and Reactome pathways are shown in Table S9-S11. These DEPs mainly belong to immunity and metabolism related protein. KEGG pathway showed that they were associated with proteasome, ubiquitin mediated proteolysis, protein processing in endoplasmic reticulum, necroptosis, and nervous system diseases include Parkinson's disease (PD), Alzheimer's disease, amyotrophic lateral sclerosis, and Huntington disease (Fig. 4C-G).

\section{The results of plasma metabolomics analysis}

Subsequently, we conducted plasma metabolomics studies with the 5 children with ASD and their corresponding controls. In the positive ion mode of MS analysis, 9281 mass spectrum peaks were identified. The PCA analysis established that the cases and controls could not well be distinguished, although except for a gene, other cases and controls have aggregation trend (Fig. 5A). PLS-DA analysis revealed that the cases and controls could be distinguished (Fig. 5B). However, this model was a bit overfitting (Fig. 5C). It may be due to individual heterogeneity or small sample size. Likewise, 286 metabolites were identified as differential metabolites between the cases and controls (Fig. 5D, Table S12). Compared with the controls, 157 of them were increased and 129 were decreased. They could well distinguish the case group from the controls (Fig. 5E), and were mainly related to niacin and niacinamide metabolism, vitamin B6 metabolism, arginine biosynthesis, and sphingolipid metabolism (Fig. 5F). The pathways associated with the differential metabolites are shown in Table S13.

In the negative ion mode, 6989 mass spectrum peaks were identified. PCA analysis exhibited that the case group was not well distinguished from the controls. The case group showed a tendency to be more concentrated, while the control group was more dispersed among different individuals (Fig. 6A). PLS-DA analysis separated the two groups effectively, however, this model was also a bit overfitting (Fig. 6B and C). Meanwhile, 190 metabolites were identified as differential metabolites between the cases and the controls. Compared with the controls, 98 were increased and 92 were decreased for controls (Fig. 6D, Table S14). They could effectively discern the cases from the control group (Fig. 6E), and were mainly 
involved in alanine, aspartic acid and glutamate metabolism, D-glutamine and D-glutamate metabolism, arginine biosynthesis, glycerophospholipid metabolism, and nitrogen metabolism (Fig. 6F, Table S15).

\section{The integration analysis of DEPs and differential metabolites}

By integration analysis, two pathways associated with DEPs and differential metabolites identified in this study were enriched, including glycerophospholipid metabolism (Fig. 7A) and N-Glycan biosynthesis (Fig. 7B).

\section{Discussion}

In this study, we identified five de novo mutational genes associated with five ASD children among 122 clinically diagnosed autistic children. This is similar to results from previous studies of coding-sequence mutations making up $5-10 \%$ of ASD patients ${ }^{3}$. These genes include ASH1L, SCN2A, GIGYF2, NAA15, and DDX3X. They has been reported to be associated with ASD in the previous studies ${ }^{21-27}$. We then carried out plasma, PBMCs proteomics, and plasma metabolomics studies in these ASD children and healthy controls. The results showed that total plasma and PBMCs proteins, total plasma metabolites, were not well distinguished between the two groups, but DEPs and differential metabolites between these two groups were able to distinguish the two groups. Here, we focus on these DEPs, differential metabolites, and their related mechanisms.

By plasma proteomics analysis, 13 DEPs were identified. Consistent with the previous studies, these DEPs identified in the blood were mainly involved in complement and coagulation cascades, inflammatory and immune responses. Altered complement proteins in the blood from patients with ASD have been broadly reported, including changes in protein expression levels ${ }^{15,28-31}$ and post-translational modifications ${ }^{32,33}$. Complement $\mathrm{C} 5$ was also found to be up-regulated in the PBMCs of children with ASD in our previous study ${ }^{11}$. Here, C1QA, C1QB, C1S, and C2 were significantly up-regulated in the plasma of children with ASD, whereas COLEC10 was down-regulated. To the best of our knowledge, C1S, C2, and COLEC10 were first reported to be associated with ASD. The expression of most complement proteins is up-regulated in the periphery of autistic patients, suggesting that the complement pathway may be activated in the periphery of autistic patients. However, the expression trends of complement proteins in the brain of autistic patient are not completely consistent ${ }^{34,35}$. On the other hand, beyond its involvement with innate immune responses, complement proteins has been increasingly implicated in playing an important role in neurodevelopment, including neurogenesis, neuronal migration, and synaptic remodeling ${ }^{34-36}$. Together, the changes in the complement system in peripheral blood, PBMCs, and brain of ASD patients highlights that this system may play a key role in the pathogenesis of ASD and is worthy of further study. 
Accumulating evidence suggests a potential role of the immune system in the pathophysiology of ASD during the pre, neo-, and postnatal periods ${ }^{13,37}$. In this study, 7 plasma DEPs and 36 PBMCs DEPs were involved in the immune system, further supporting the association between immunity and ASD pathogenesis. Ten DEPs of PBMCs were associated with interleukin-1 (IL-1), together with our previous study (IL-12) ${ }^{11}$ and other studies ${ }^{38,39}$, suggesting that IL-1 and IL-12 might be key inflammatory cytokines in peripheral blood and blood mononuclear cells.

Mitochondrial dysfunction has also been implicated in immune dysregulation in ASD ${ }^{40}$. In this study, the expression of three proteins (NDUFA7, NDUFA11, and NDUFA13) related to energy metabolism were increased in PBMCs of autistic children. These results were similar to previous observations in PBMCs ${ }^{11}$ and LCL cells ${ }^{41}$, different tissues and organs ${ }^{42}$, T cell, NK cell, and monocyte ${ }^{43}$ in children with autism. Activation and proliferation of microglia and astrocytes have also been observed in the brains of ASD subjects ${ }^{44}$. Interestingly, transcriptome analysis between autistic brains and normal brains identified discrete modules by gene co-expression network analysis: a neuronal module and a module enriched for immune genes and glial markers ${ }^{45}$. This may explain the uniqueness of mitochondrial dysfunction in ASD and how autistic children may have nontraditional mitochondrial diseases. After all, mitochondrial dysfunction has been reported by postmortem brain tissue examinations on ASD subjects and reduction of ETC (Electron transport chain) complexes has also been observed in different brain regions of autistic children ${ }^{46-48}$.

Consistent with our previous study ${ }^{11}$, the main pathways associated with DEPs of PBMCs have also involved in proteasome, ubiquitin mediated proteolysis, and protein processing in the endoplasmic reticulum, which strongly suggest that endoplasmic reticulum stress (ER) being linked to the PBMCs of children with ASD. ER stress occurs when the amount of unfolded proteins in the ER reach an unmanageable level, triggering the unfolded protein response (UPR) ${ }^{49}$. Under excess or chronic ER stress, cell apoptosis is induced to eliminate unhealthy cells ${ }^{50}$. Here, 6 DEPs were associated with necroptosis. Similarly, LCLs from children with autism were shown to be more sensitive to necrosis than their nonautistic siblings ${ }^{51}$. Thus, necroptosis may be associated with the ER stress in the PBMCs of ASD subjects. Moreover, increase in ER stress has been observed in the brains of autistic children ${ }^{52,53}$ and those of ASD model mice ${ }^{54}$. The mutation of ASD related genes and the aggregation of their encoded proteins ${ }^{11,52}$ and oxidative stress may be responsible for ER stress in children with ASD ${ }^{52}$. On the other hand, the proteostasis network machinery plays a role in the establishment, maintenance, and plasticity of stable and dynamic dendritic arbors. Ubiquitin-proteasome system is required for developmental dendritic pruning ${ }^{55,56}$.

Interestingly, among the DEPs of PBMS, three other genes have also been linked to ASD in addition to DDX3X, including SLC9A $9{ }^{57}, \mathrm{CSDE}^{15}$, and CCT $4^{59}$. Among them, The SLC9A9 gene encodes $\mathrm{Na}^{+} / \mathrm{H}^{+}$ transpteron-9 endometrium protein (NHE9), which has been shown to be related to endocytosis, protein ubiquitination, and phagosome ${ }^{57}$. CSDE1 encodes RNA-binding proteins that may be involved in 
translation-coupled mRNA conversion and is associated with neurodevelopment and neuropsychiatric disorders ${ }^{58}$. The CCT4 gene encodes a molecular chaperone that assists in protein folding during ATP hydrolysis ${ }^{60}$. These results further support that protein folding and ER stress may be associated with the pathogenesis of ASD, and that at least some children with ASD carry more than two risk genes in this study.

By metabolomics analysis, the differential metabolites in the plasma between cases and controls were mainly involved in amino acid (alanine, aspartate and glutamate, arginine biosynthesis, and D-glutamine and D-glutamate), vitamin (nicotinate and nicotinamide, and vitamin B6), and lipid (glycerophospholipid and sphingolipid) metabolism. Nitrogen metabolism, $\mathrm{N}$-Glycan biosynthesis, and neomycin, kanamycin and gentamicin biosynthesis were also involved.

In the present study, among the altered amino acids, L-Glutamate was down-regulated in plasma of controls while L-Glutamine was increased in plasma of autistic children. Although previous studies showed that the altered levels of glutamate and glutamine in the blood of autistic patients were inconsistent ${ }^{61-66}$, these data support the current view that excitatory/inhibitory imbalance, especially the abnormality of the excitatory neurotransmitter glutamate, is one of the pathogenesis of ASD ${ }^{65,67}$. Indeed, it has been reported that glutamate signals in the anterior cingulate cortex and cerebellum of ASD patients were significantly decreased ${ }^{68}$. More recently, the gut metabolites involved in alanine, aspartate, and glutamate metabolic pathways were reported to be significantly lower in children with ASD, which was associated with differences in the abundance of gut microbiota related to D-Glutamine and Dglutamate metabolism, suggesting that the gut microbiota might contribute to abnormal glutamate metabolism in autistic children ${ }^{69}$. Besides, similar with the present study, aspartate has been observed to be decreased in the fecal ASD subjects ${ }^{70}$, while ornithine was observed to be increased in the blood of children with $\mathrm{ASD}^{64}$.

Our results showed that three differential metabolites were also involved in the nicotinate and nicotinamide metabolism pathway. Niacin (NA), also known as Vitamin B3 and nicotinic acid, can be biosynthetically converted into nicotinamide adenine dinucleotide (NAD). NAD has a variety of biological functions and plays a central role in redox reactions ${ }^{71}$. Nicotinate and nicotinamide metabolism have been reported to be altered in the prefrontal cortex ${ }^{72}$, urine ${ }^{73}$, and blood ${ }^{74}$ of ASD individuals, and associated with microbiota transfer therapy (MTT) of autistic children ${ }^{74}$. Nicotinamide is derived from tryptophan, while abnormal tryptophan metabolism has been observed in children with $\mathrm{ASD}^{73,75-77}$. In addition, three differential metabolites (O-Phospho-4-hydroxy-L-threonine, Pyridoxamine, and 4Pyridoxate) involved in Vitamin B6 metabolism were found to be decreased in autistic children. Reduced levels of Vitamin B6 have been observed in the urine of autistic children ${ }^{78}$. It is the main cofactor of biological reactions and is important for the synthesis of neurotransmitters and trans-sulfuration. Its deficiency is related to oxidative stress, high blood homocysteine and hypomethylation in children with $A D^{78}$. In general, the lack of vitamin $B$ group in children with ASD may be caused by nutritional deficiency, poor absorption or alteration in gut microbiota ${ }^{74,79,80}$, contributing to the pathogenesis of ASD. 
The brain is particularly enriched in lipids with a diverse lipid composition compared to other tissues ${ }^{81}$. Glycerophospholipids are critical components of neuronal membranes and myelin, and principal regulators of synaptic function ${ }^{81}$. Sphingolipid is involved in neuronal differentiation, synaptic transmission in neuronal-glial connections, and myelin stability. Disturbance of their metabolism has been linked to various neuropsychiatric diseases include autism ${ }^{82}$, Rett syndrome ${ }^{83}$, and ASD $^{84}$.

Finally, integrating omics data, glycerophospholipid and N-linked glycosylation metabolism was associated with the DEPs and differential metabolites. N-linked glycosylation is important in brain structure and function. The extracellular glycans and glycoconjugates may contribute to the etiology and pathogenesis of pervasive neurodevelopmental disorders include idiopathic ASDs. Glycobiology related genes were implicated in ASD. Mutations in glycogenes associated with ASD affect the downstream steps of N-glycan biosynthesis ${ }^{85}$.

\section{Limitations}

Although we detected 122 children in the genetic testing, and the rate of children carrying risk genes is also consistent with the previous report ${ }^{3}$, the rate itself is low. Thus, the number of samples carrying risk genes used in omics studies is limited, and further research with a large sample size is required. Secondly, in further research, it is also interesting to add a group in which that the children were diagnosed as ASD, but not to be detected to carry risk gene.

\section{Conclusions}

In this study, our results showed that although there was heterogeneity at the genetic level in children with ASD, the DEPs of plasma and PBMCs and differential metabolites of plasma could still distinguish the cases from controls. The proteomic results highlighted the roles of complement, inflammation and immunity, mitochondrial dysfunction, proteasome, ubiquitin mediated proteolysis, and ER stress in the pathogenesis of ASD. Metabolomic results mainly showed the disturbances of amino acid, vitamin, and lipid metabolism in children with ASD (Fig. 8). The mechanisms and pathways associated with the DEPs and differential metabolites have been reported in previous studies. The results agreed with the view that children with ASD might have an important underlying common mechanism. They are not only potential therapeutic targets for ASD but also significant contributors for studying biomarkers for the disorder $1,6-$ 8,13 .

\section{Abbreviations}

ASD

autism spectrum disorder

$\mathrm{BP}$

biological process

CC 
cellular component

DEPS

differentially expressed proteins

ER

endoplasmic reticulum stress

iTRAQ

isobaric tagging reagents for relative and absolute quantification

MS

mass spectrometry

PLS-DA

orthogonal projections to latent structures-discriminant analysis

PBMCs

peripheral blood mononuclear cells

PCA

principal component analysis

VIP

variable importance in projection.

\section{Declarations}

\section{Acknowledgments}

We thank all the individuals who participated in the study and the Instrument Analysis Center of Shenzhen University.

\section{Authors' contributions}

LMS and CYF. conceived and designed the experiments; HJZ, JL, XKL, YXZ, and XXT performed the experiments and analyzed the data; LMS and XSC wrote the manuscript. MC, NUK, and XSC reviewed and edited the manuscript. YG, CYF, and QH carried out the clinical studies and collected the blood samples. All authors read and approved the final manuscript.

\section{Funding}

This study was financially supported by National Natural Science Foundation of China (No. 31870825), and the Shenzhen Bureau of Science, Technology, and Information (No. JCYJ20170412110026229), and Shenzhen-Hong Kong Institute of Brain Science-Shenzhen Fundamental Research Institutions (No. 2021SHIBS0003).

\section{Availability of data and materials}

The datasets generated and/or analyzed during the current study are available from the corresponding author on reasonable request. 


\section{Declarations}

\section{Ethics approval and consent to participate}

The Maternal and Child Health Hospital of Baoan, and the Shenzhen University approved study procedures. The experiments were performed after obtaining written consent from caretakers of the children under observation according to the guidelines of this hospital.

\section{Consent for publication}

Not applicable.

\section{Competing interest}

The authors declare that they have no competing interests.

\section{Author details}

${ }^{1}$ College of Life Science and Oceanography, Shenzhen University, Shenzhen 518060, P. R. China.

${ }^{2}$ Maternal and Child Health Hospital of Baoan, Shenzhen 518100, P. R. China. ${ }^{3}$ Department of Psychology, Emory University, Atlanta, GA 30322, USA. ${ }^{4}$ Shenzhen-Hong Kong Institute of Brain ScienceShenzhen Fundamental Research Institutions, Shenzhen 518055, P. R. China. ${ }^{5}$ Brain Disease and Big Data Research Institute, Shenzhen University, Shenzhen 518071, P. R. China.

\section{References}

1. Shen L, et al. Biomarkers in autism spectrum disorders: Current progress. Clin Chim Acta Int J Clin Chem. 2020;502:41-54.

2. El-Fishawy P, State MW. The Genetics of Autism: Key Issues, Recent Findings and Clinical Implications. Psychiatr Clin North Am. 2010;33:83-105.

3. Huguet G, Ey E, Bourgeron T. The genetic landscapes of autism spectrum disorders. Annu Rev Genomics Hum Genet. 2013;14:191-213.

4. Eldevik S, et al. Meta-analysis of Early Intensive Behavioral Intervention for children with autism. J Clin Child Adolesc Psychol Off J Soc Clin Child Adolesc Psychol Am Psychol Assoc Div. 2009;53 38:439-50.

5. Chen $L$, et al. Oxidative stress marker aberrations in children with autism spectrum disorder: a systematic review and meta-analysis of 87 studies ( $N=9109)$. Transl Psychiatry. 2021;11:15.

6. Shen L, et al. Advances in Biomarker Studies in Autism Spectrum Disorders. Adv Exp Med Biol. 2019;1118:207-33.

7. Ristori MV, et al. Proteomics and Metabolomics Approaches towards a Functional Insight onto AUTISM Spectrum Disorders: Phenotype Stratification and Biomarker Discovery. Int. J. Mol. Sci. 21, (2020). 
8. Park DI. Genomics, transcriptomics, proteomics and big data analysis in the discovery of new diagnostic markers and targets for therapy development. Prog Mol Biol Transl Sci. 2020;173:61-90.

9. Diagnostic and statistical manual of mental disorders: DSM-5. (American Psychiatric Association, 2013).

10. Liu H, et al. Biomarker Exploration in Human Peripheral Blood Mononuclear Cells for Monitoring Sulforaphane Treatment Responses in Autism Spectrum Disorder. Sci Rep. 2020;10:5822.

11. Shen L, et al. Proteomics Study of Peripheral Blood Mononuclear Cells (PBMCs) in Autistic Children. Front Cell Neurosci. 2019;13:105.

12. Frye RE, et al. Redox metabolism abnormalities in autistic children associated with mitochondrial disease. Transl Psychiatry. 2013;3:e273-3.

13. Masi A, DeMayo MM, Glozier N, Guastella AJ. An Overview of Autism Spectrum Disorder, Heterogeneity and Treatment Options. Neurosci Bull. 2017;33:183-93.

14. Nuttle X, Itsara A, Shendure J, Eichler EE. Resolving genomic disorder-associated breakpoints within segmental DNA duplications using massively parallel sequencing. Nat Protoc. 2014;9:1496-513.

15. Shen L, et al. iTRAQ-Based Proteomic Analysis Reveals Protein Profile in Plasma from Children with Autism. PROTEOMICS - Clin Appl. 2018;12:1700085.

16. OMICSBEAN website. http://www.omicsbean.cn. Accessed 20 September 2021.

17. Shen L, et al. Proteomic Profiling of Cerebrum Mitochondria, Myelin Sheath, and Synaptosome Revealed Mitochondrial Damage and Synaptic Impairments in Association with $3 \times$ Tg-AD Mice Model. Cell Mol Neurobiol. 2021. doi:10.1007/s10571-021-01052-z.

18. MetaboAnalyst. 4.0, https://www.metaboanalyst.ca/. Accessed 20 September 2021.

19. Search Tool for the Retrieval of Interacting Genes/Proteins,http://string-db.org/. Accessed 20 September 2021.

20. Karnovsky A, et al. Metscape 2 bioinformatics tool for the analysis and visualization of metabolomics and gene expression data. Bioinformatics. 2012;28:373-80.

21. Bagnell A. The Role of ASH1L During Human Neurodevelopment. Sr. Theses 1-53 (2019).

22. Begemann A, et al. Further corroboration of distinct functional features in SCN2A variants causing intellectual disability or epileptic phenotypes. Mol Med Camb Mass. 2019;25:6.

23. Cheng $\mathrm{H}$, et al. Truncating Variants in NAA15 Are Associated with Variable Levels of Intellectual Disability, Autism Spectrum Disorder, and Congenital Anomalies. Am J Hum Genet. 2018;102:98594.

24. Gazzellone MJ, et al. Copy number variation in Han Chinese individuals with autism spectrum disorder. J Neurodev Disord. 2014;6:34.

25. Garcia-Forn M, Boitnott A, Akpinar Z, De Rubeis S. Linking Autism Risk Genes to Disruption of Cortical Development. Cells 9, (2020).

26. Wang X, et al. Phenotypic expansion in DDX3X - a common cause of intellectual disability in females. Ann Clin Transl Neurol. 2018;5:1277-85. 
27. Iossifov I, et al. The contribution of de novo coding mutations to autism spectrum disorder. Nature. 2014;515:216-21.

28. Momeni N, et al. A novel blood-based biomarker for detection of autism spectrum disorders. Transl Psychiatry. 2012;2:e91-1.

29. Corbett BA, et al. A proteomic study of serum from children with autism showing differential expression of apolipoproteins and complement proteins. Mol Psychiatry. 2007;12:292-306.

30. Warren RP. Decreased Plasma Concentrations of the C4B Complement Protein in Autism. Arch Pediatr Adolesc Med. 1994;148:180.

31. Hewitson L, et al. Blood biomarker discovery for autism spectrum disorder: A proteomic analysis. PloS One. 2021;16:e0246581.

32. Feng $\mathrm{C}$, et al. Redox proteomic identification of carbonylated proteins in autism plasma: insight into oxidative stress and its related biomarkers in autism. Clin. Proteomics 14, (2017).

33. Qin Y, et al. Serum glycopattern and Maackia amurensis lectin-II binding glycoproteins in autism spectrum disorder. Sci Rep. 2017;7:46041.

34. Magdalon J, et al. Complement System in Brain Architecture and Neurodevelopmental Disorders. Front Neurosci. 2020;14:23.

35. Jun Tan CF. C1q as a Regulator of Brain Development: Implications for Autism Spectrum Disorders. Brain Disord. Ther. 04, (2015).

36. Stephan AH, Barres BA, Stevens B. The complement system: an unexpected role in synaptic pruning during development and disease. Annu Rev Neurosci. 2012;35:369-89.

37. Meltzer A, Van de Water J. The Role of the Immune System in Autism Spectrum Disorder. Neuropsychopharmacol Off Publ Am Coll Neuropsychopharmacol. 2017;42:284-98.

38. Zimmerman AW, et al. Cerebrospinal fluid and serum markers of inflammation in autism. Pediatr Neurol. 2005;33:195-201.

39. Molloy CA, et al. Elevated cytokine levels in children with autism spectrum disorder. J Neuroimmunol. 2006;172:198-205.

40. Frye RE. Mitochondrial Dysfunction in Autism Spectrum Disorder: Unique Abnormalities and Targeted Treatments. Semin Pediatr Neurol. 2020;35:100829.

41. Rose $S$, et al. Mitochondrial and redox abnormalities in autism lymphoblastoid cells: a sibling control study. FASEB J. 2017;31:904-9.

42. Bennuri SC, Rose S, Frye RE. Mitochondrial Dysfunction Is Inducible in Lymphoblastoid Cell Lines From Children With Autism and May Involve the TORC1 Pathway. Front Psychiatry. 2019;10:269.

43. Mead J, Ashwood P. Evidence supporting an altered immune response in ASD. Immunol Lett. 2015;163:49-55.

44. Liao X, Yang J, Wang H, Li Y. Microglia mediated neuroinflammation in autism spectrum disorder. J Psychiatr Res. 2020;130:167-76. 
45. Voineagu I, et al. Transcriptomic analysis of autistic brain reveals convergent molecular pathology. Nature. 2011;474:380-4.

46. Carrasco M, Salazar C, Tiznado W, Ruiz LM. Alterations of Mitochondrial Biology in the Oral Mucosa of Chilean Children with Autism Spectrum Disorder (ASD). Cells. 2019;8:367.

47. Chauhan $A$, et al. Brain region-specific deficit in mitochondrial electron transport chain complexes in children with autism. J Neurochem. 2011;117:209-20.

48. Tang G, et al. Mitochondrial abnormalities in temporal lobe of autistic brain. Neurobiol Dis. 2013;54:349-61.

49. Martínez G, Khatiwada S, Costa-Mattioli M, Hetz C. ER Proteostasis Control of Neuronal Physiology and Synaptic Function. Trends Neurosci. 2018;41:610-24.

50. Takasugi N, et al. The Emerging Role of Electrophiles as a Key Regulator for Endoplasmic Reticulum (ER) Stress. Int. J. Mol. Sci. 20, (2019).

51. Main PAE, Thomas P, Esterman A, Fenech MF. Necrosis is increased in lymphoblastoid cell lines from children with autism compared with their non-autistic siblings under conditions of oxidative and nitrosative stress. Mutagenesis. 2013;28:475-84.

52. Dong D, Zielke HR, Yeh D, Yang P. Cellular stress and apoptosis contribute to the pathogenesis of autism spectrum disorder: The role of cellular stress and apoptosis in autism. Autism Res. 2018;11:1076-90.

53. Crider A, Ahmed AO, Pillai A. Altered Expression of Endoplasmic Reticulum Stress-Related Genes in the Middle Frontal Cortex of Subjects with Autism Spectrum Disorder. Mol Neuropsychiatry. 2017;3:85-91.

54. Kawada $\mathrm{K}$, et al. Aberrant neuronal differentiation and inhibition of dendrite outgrowth resulting from endoplasmic reticulum stress. J Neurosci Res. 2014;92:1122-33.

55. Lottes EN, Cox DN. Homeostatic Roles of the Proteostasis Network in Dendrites. Front. Cell. Neurosci. $14,(2020)$.

56. Hamilton AM, Zito K Breaking it down: the ubiquitin proteasome system in neuronal morphogenesis. Neural Plast. 2013, 196848 (2013).

57. Zhang-James Y, et al. Effect of disease-associated SLC9A9 mutations on protein-protein interaction networks: implications for molecular mechanisms for ADHD and autism. Atten Deficit Hyperact Disord. 2019;11:91-105.

58. Guo $\mathrm{H}$, et al. Disruptive variants of CSDE1 associate with autism and interfere with neuronal development and synaptic transmission. Sci Adv. 2019;5:eaax2166.

59. lossifov I, et al. The contribution of de novo coding mutations to autism spectrum disorder. Nature. 2014;515:216-21.

60. Sergeeva OA, et al. Human CCT4 and CCT5 chaperonin subunits expressed in Escherichia coli form biologically active homo-oligomers. J Biol Chem. 2013;288:17734-44. 
61. Shinohe A, et al. Increased serum levels of glutamate in adult patients with autism. Prog Neuropsychopharmacol Biol Psychiatry. 2006;30:1472-7.

62. Shimmura $C$, et al. Alteration of plasma glutamate and glutamine levels in children with highfunctioning autism. PloS One. 2011;6:e25340.

63. Delaye J-B, et al. Post hoc analysis of plasma amino acid profiles: towards a specific pattern in autism spectrum disorder and intellectual disability. Ann Clin Biochem Int J Lab Med. 2018;55:54352.

64. Smith AM, et al. Amino Acid Dysregulation Metabotypes: Potential Biomarkers for Diagnosis and Individualized Treatment for Subtypes of Autism Spectrum Disorder. Biol Psychiatry. 2018. doi:10.1016/j.biopsych.2018.08.016.

65. Al-Otaish $\mathrm{H}$, et al. Relationship between absolute and relative ratios of glutamate, glutamine and GABA and severity of autism spectrum disorder. Metab Brain Dis. 2018;33:843-54.

66. Zheng Z, Zhu T, Qu Y, Mu D. Blood Glutamate Levels in Autism Spectrum Disorder: A Systematic Review and Meta-Analysis. PloS One. 2016;11:e0158688.

67. Horder $\mathrm{J}$, et al. Glutamate and GABA in autism spectrum disorder-a translational magnetic resonance spectroscopy study in man and rodent models. Transl Psychiatry. 2018;8:106.

68. Tebartz van Elst, L, et al. Disturbed cingulate glutamate metabolism in adults with high-functioning autism spectrum disorder: evidence in support of the excitatory/inhibitory imbalance hypothesis. Mol Psychiatry. 2014;19:1314-25.

69. Wang M, et al Alterations in Gut Glutamate Metabolism Associated with Changes in Gut Microbiota Composition in Children with Autism Spectrum Disorder. mSystems 4, (2019).

70. Kang D-W, et al. Differences in fecal microbial metabolites and microbiota of children with autism spectrum disorders. Anaerobe. 2018;49:121-31.

71. Doroftei B, et al. Minireview Exploring the Biological Cycle of Vitamin B3 and Its Influence on Oxidative Stress: Further Molecular and Clinical Aspects. Mol. Basel Switz. 25, (2020).

72. Kurochkin I, et al. Metabolome signature of autism in the human prefrontal cortex. Commun Biol. 2019;2:234.

73. Yap IKS, et al. Urinary Metabolic Phenotyping Differentiates Children with Autism from Their Unaffected Siblings and Age-Matched Controls. J Proteome Res. 2010;9:2996-3004.

74. Kang D-W, et al Distinct Fecal and Plasma Metabolites in Children with Autism Spectrum Disorders and Their Modulation after Microbiota Transfer Therapy. mSphere 5, (2020).

75. Gao K, Mu C-L, Farzi A, Zhu W-Y. Tryptophan Metabolism: A Link Between the Gut Microbiota and Brain. Adv Nutr Bethesda Md. 2020;11:709-23.

76. De Angelis $M$, et al. Fecal Microbiota and Metabolome of Children with Autism and Pervasive Developmental Disorder Not Otherwise Specified. PLoS ONE. 2013;8:e76993.

77. Xiao L, et al Fecal Microbiome Transplantation from Children with Autism Spectrum Disorder Modulates Tryptophan and Serotonergic Synapse Metabolism and Induces Altered Behaviors in 
Germ-Free Mice. mSystems 6, (2021).

78. Belardo A, Gevi F, Zolla L. The concomitant lower concentrations of vitamins B6, B9 and B12 may cause methylation deficiency in autistic children. J Nutr Biochem. 2019;70:38-46.

79. Barnhill K, et al. Dietary status and nutrient intake of children with autism spectrum disorder: A casecontrol study. Res Autism Spectr Disord. 2018;50:51-9.

80. Srikantha P, Mohajeri MH. The Possible Role of the Microbiota-Gut-Brain-Axis in Autism Spectrum Disorder. Int. J. Mol. Sci. 20, (2019).

81. Hussain G, et al. Role of cholesterol and sphingolipids in brain development and neurological diseases. Lipids Health Dis. 2019;18:26.

82. Wang $\mathrm{H}$, et al. Potential serum biomarkers from a metabolomics study of autism. J Psychiatry Neurosci. 2016;41:27-37.

83. Cappuccio G, et al. Sphingolipid Metabolism Perturbations in Rett Syndrome. Metabolites 9, (2019).

84. Needham BD, et al. Plasma and Fecal Metabolite Profiles in Autism Spectrum Disorder. Biol Psychiatry. 2021;89:451-62.

85. Dwyer CA, Esko JD. Glycan susceptibility factors in autism spectrum disorders. Mol Aspects Med. 2016;51:104-14.

\section{Figures}

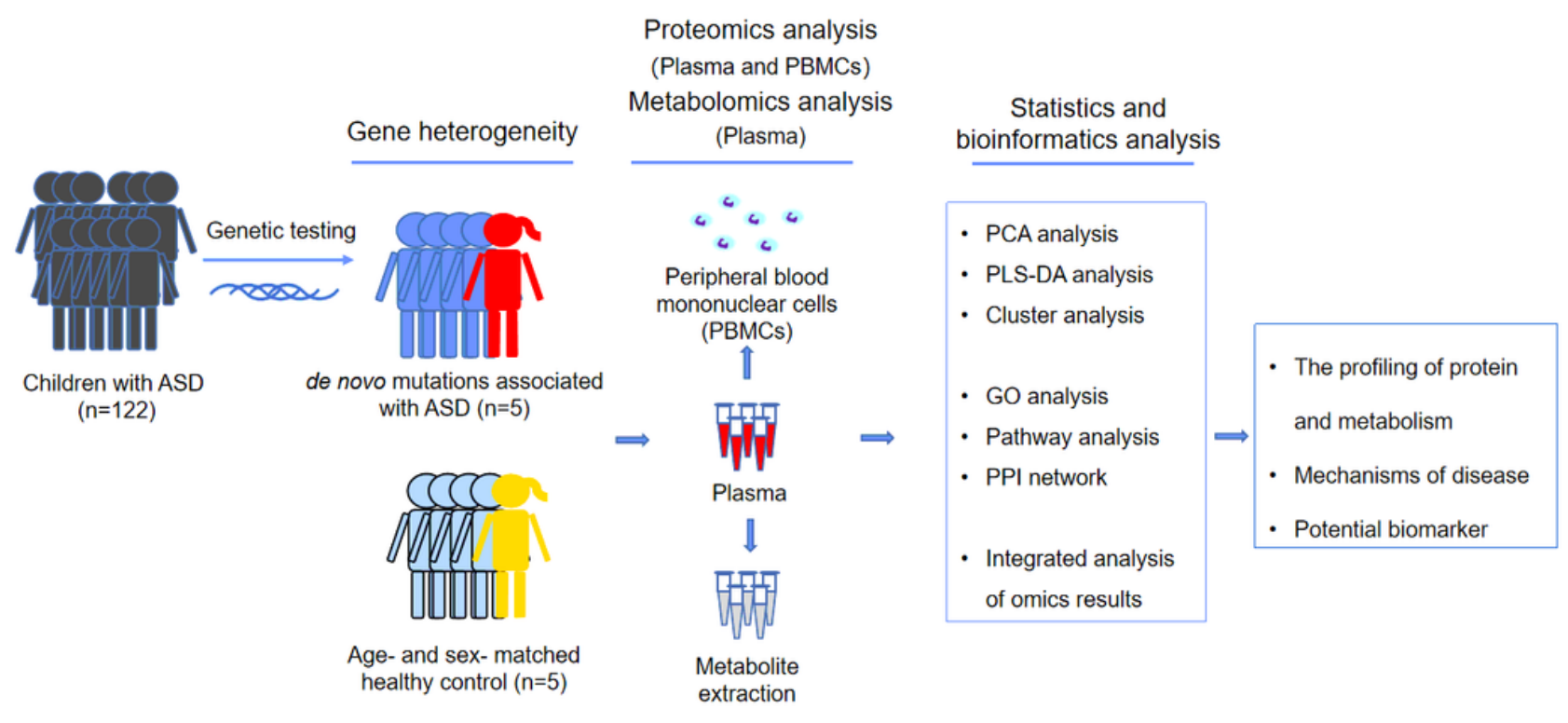

Figure 1 
Flow diagram of the study. An overview of the workflow used in this study is shown in Figure 1.

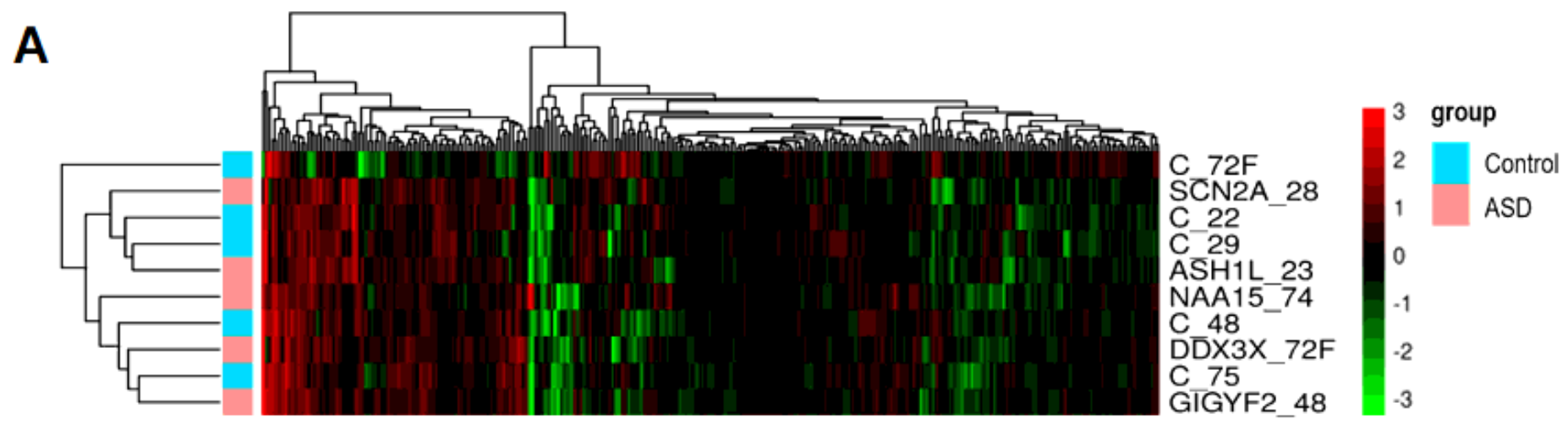

B

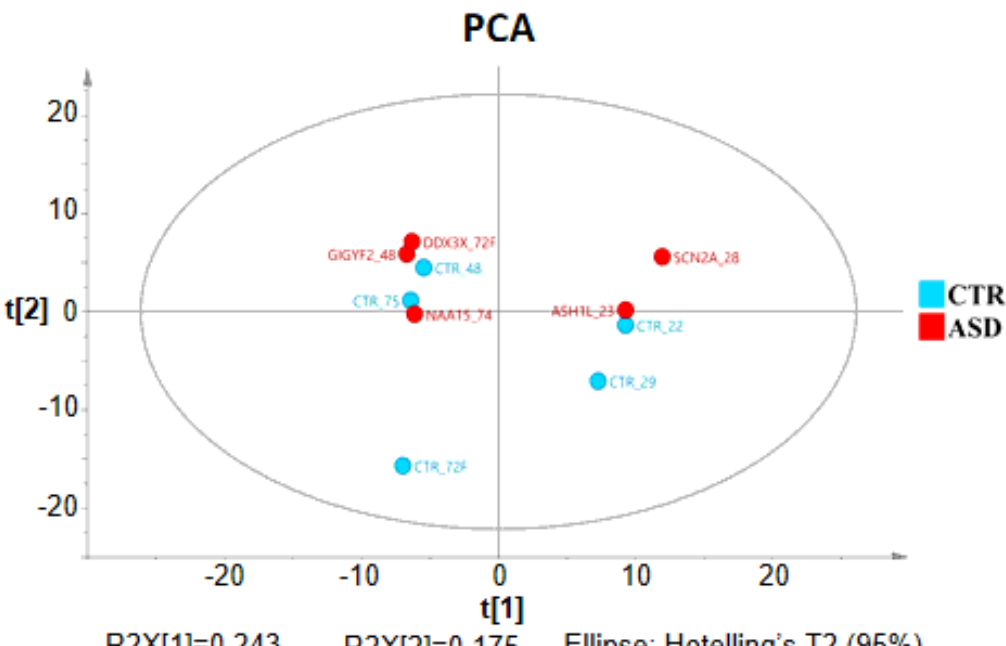

$\mathrm{R} 2 \mathrm{X}[1]=0.243 \quad \mathrm{R} 2 \mathrm{X}[2]=0.175 \quad$ Ellipse: Hotelling's T2 $(95 \%)$

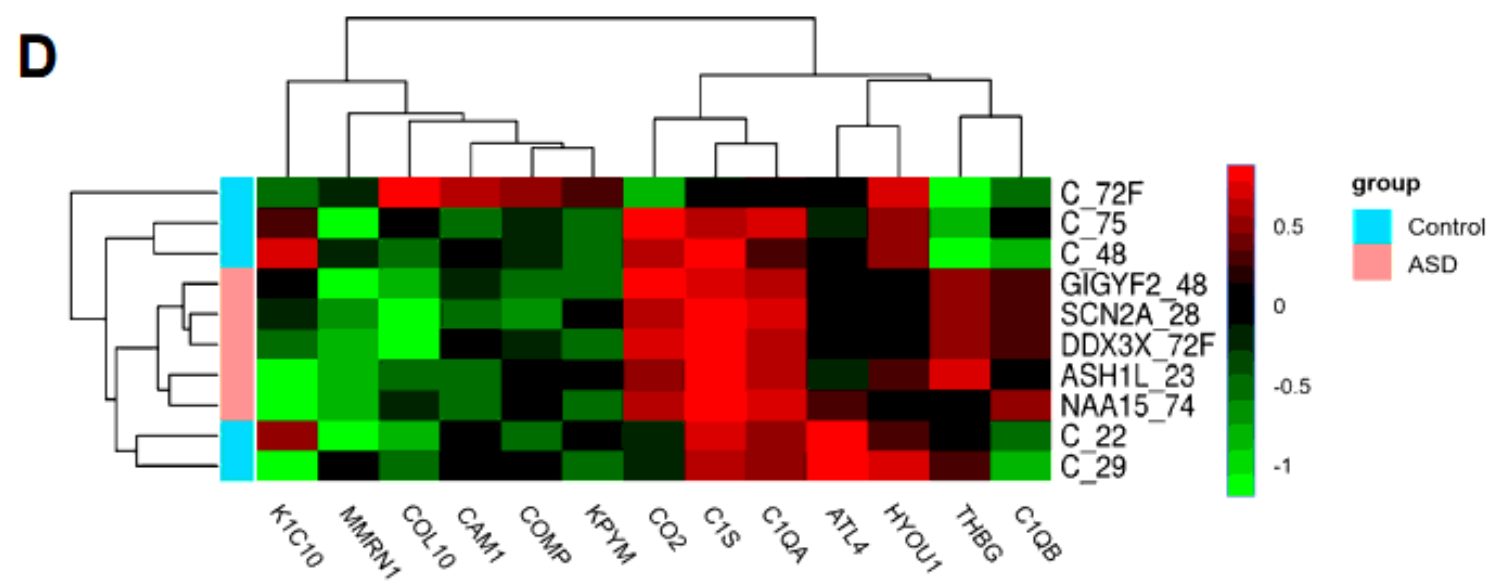

ASD-CTR

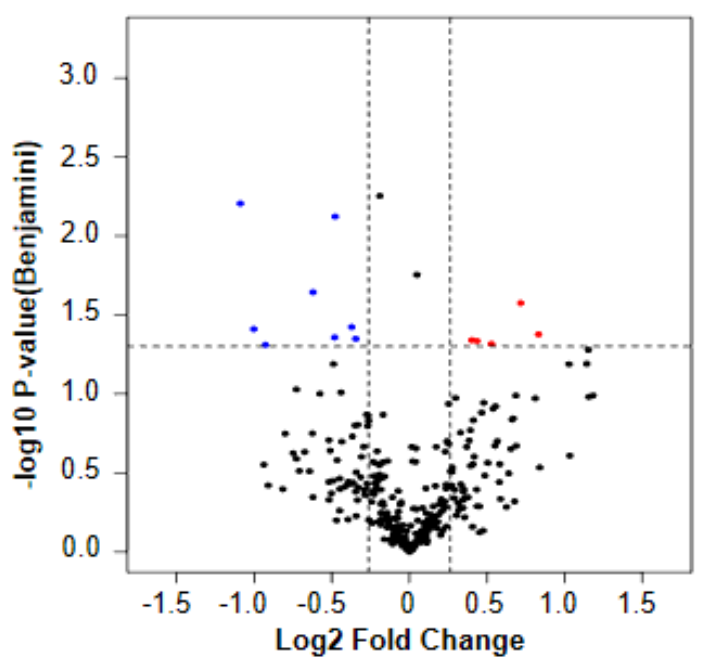

Figure 2

The DEPs identified in plasma between children with ASD and healthy controls. (A) Cluster map of plasma protein expression in the children with ASD and healthy controls. (B) PCA analysis of the plasma protein expression in children with ASD and healthy controls. (C) Volcano plots depicted the distribution of plasma proteins between children with ASD and healthy controls. The log2 fold change (FC) is plotted 
versus the $\log 10$ of the $P$ value (Benjamini). Red dots $=$ hits with $p<0.05$ and mean $\log 2 \mathrm{FC}>0.26$; blue dots=hits with $p<0.05$ and mean $|\log 2 \mathrm{FC}|<0.26$. (D) Cluster analysis of the DEPs of plasma.

A

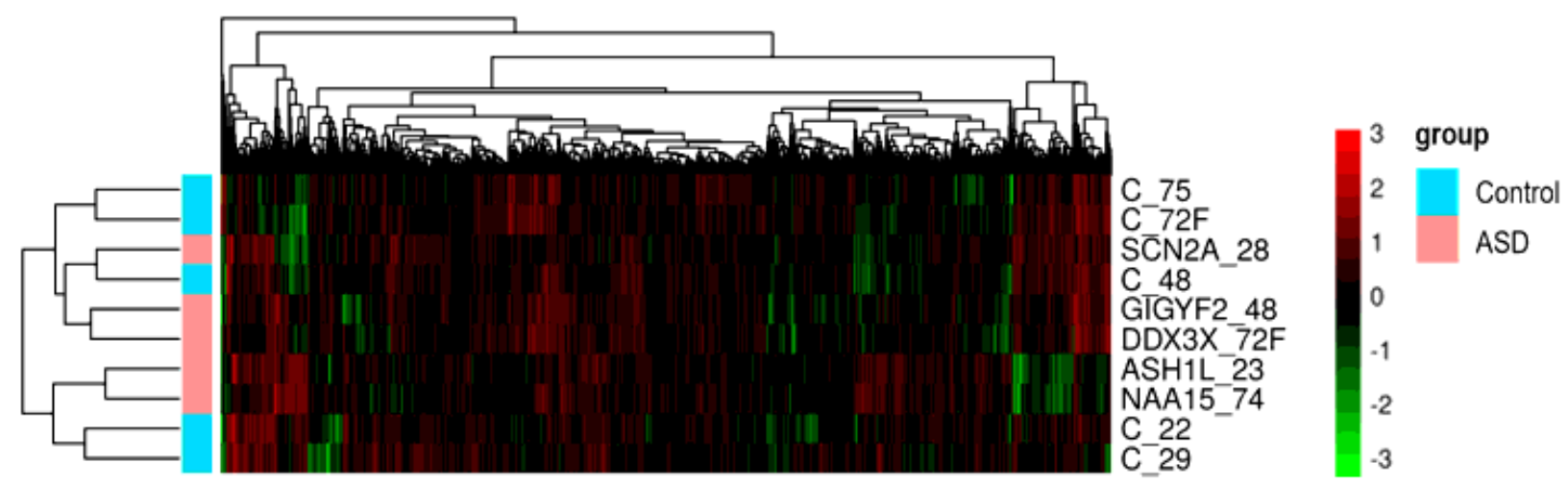

B

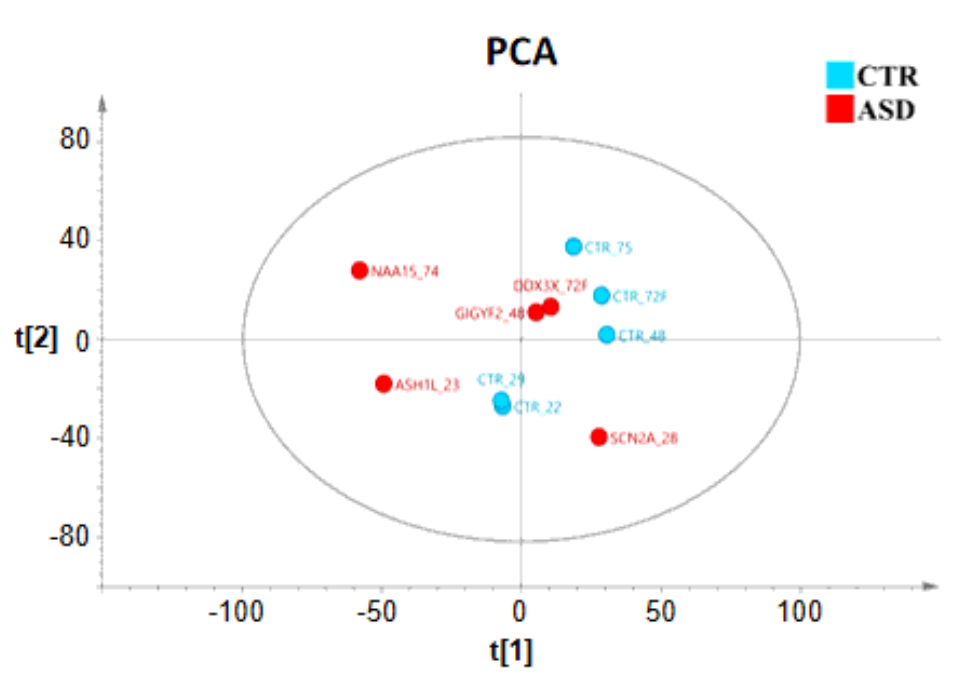

C

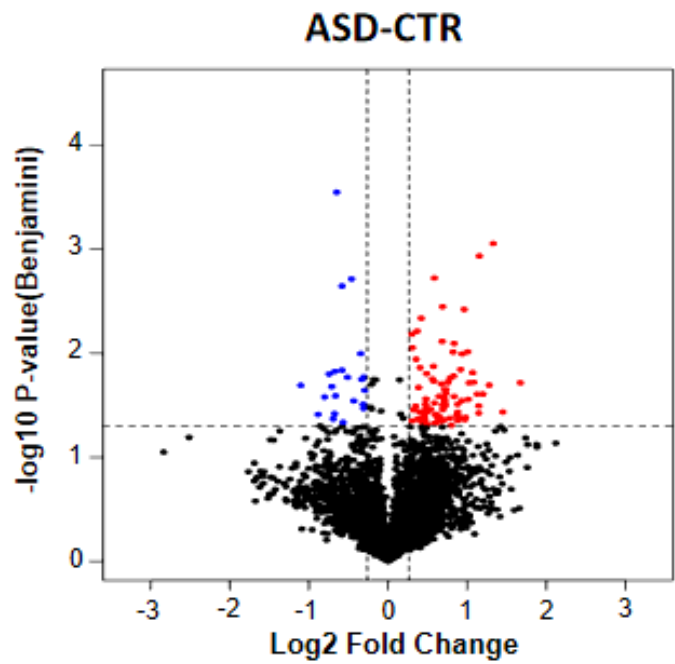

$R 2 X[1]=0.312 \quad R 2 X[2]=0.21 \quad$ Ellipse: Hotelling's T2 (95\%)

D

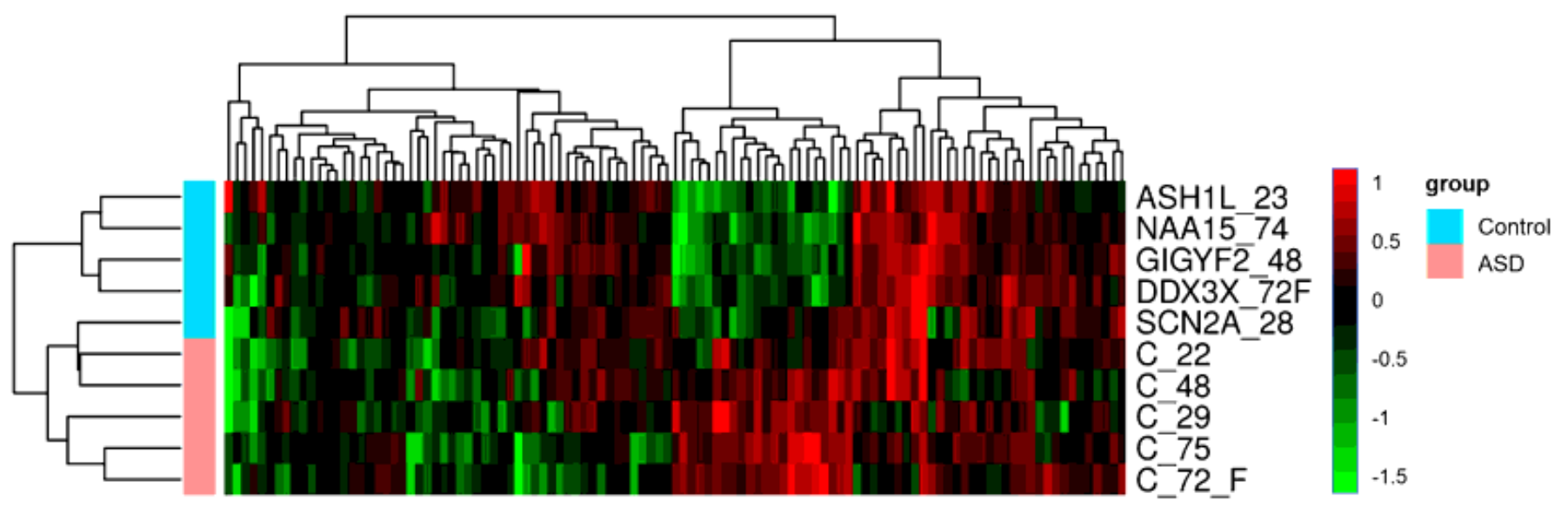

Figure 3

The DEPs identified in PBMCs between children with ASD and healthy controls. (A) Cluster map of PBMCs protein expression in the children with ASD and healthy controls. (B) PCA analysis the PBMCs protein expression in children with ASD and healthy controls. (C) Volcano plots depicted the distribution 
of PBMCs proteins between children with ASD and healthy controls. The log2 fold change (FC) is plotted versus the $-\log 10$ of the $p$-value (Benjamini). Red dots $=$ hits with $p<0.05$ and mean log2FC $>0.26$; blue dots=hits with $p<0.05$ and mean $|\log 2 F C|<0.26$. (D) Cluster analysis the DEPs of PBMCs.

A

-Log $p$ value

B

$\begin{array}{lllllll}0 & 1 & 2 & 3 & 4 & 5 & 6\end{array}$

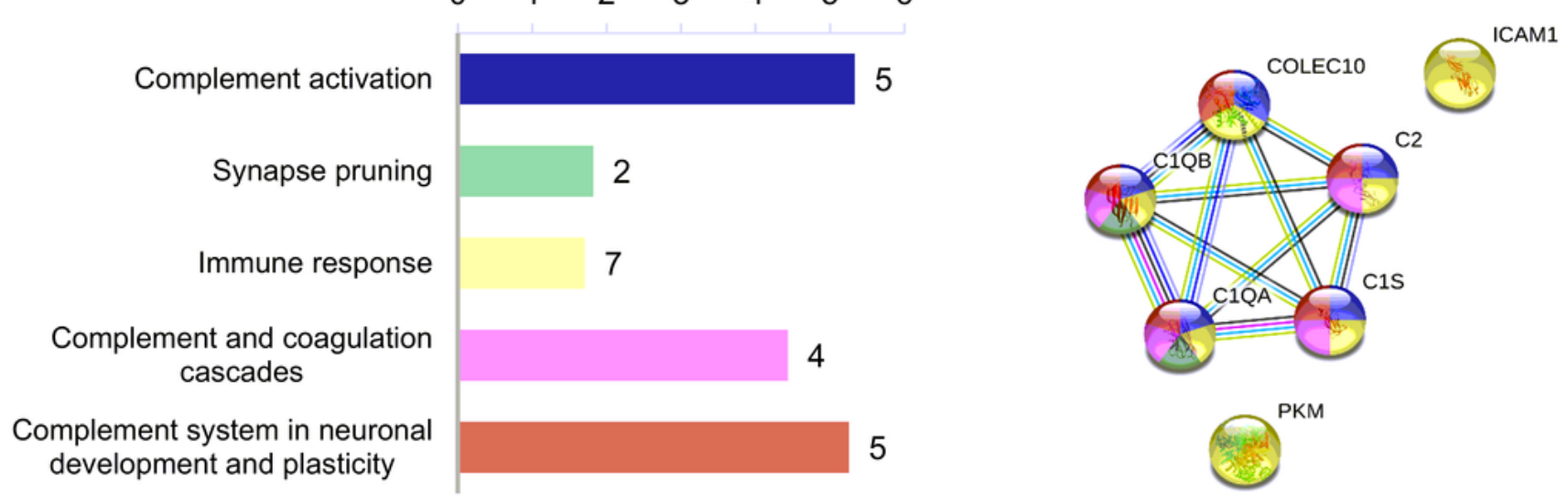

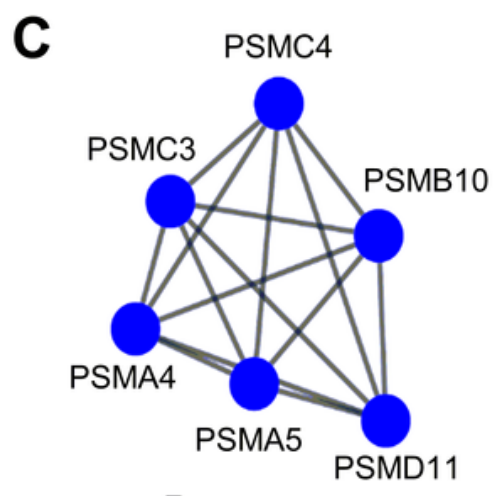

Proteasome
D

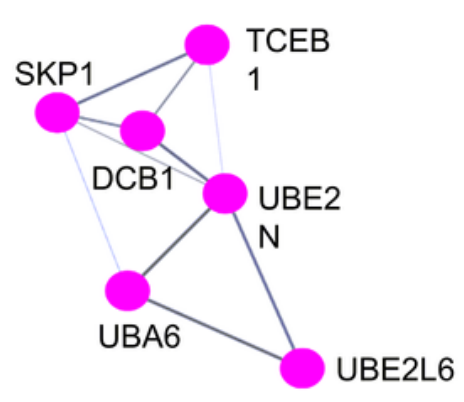

Ubiquitin mediated proteolysis

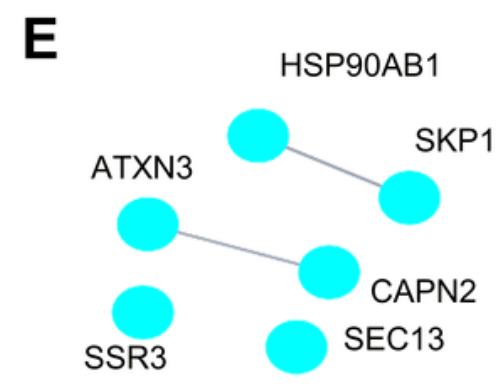

Protein processing in endoplasmic reticulum
$\mathbf{F}$

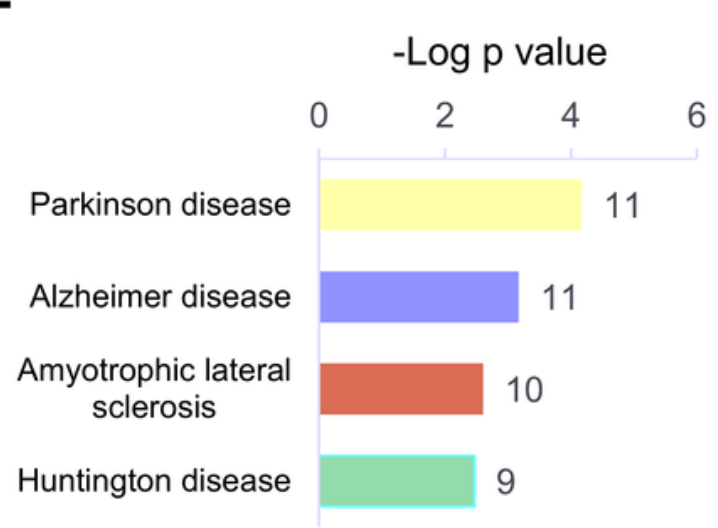

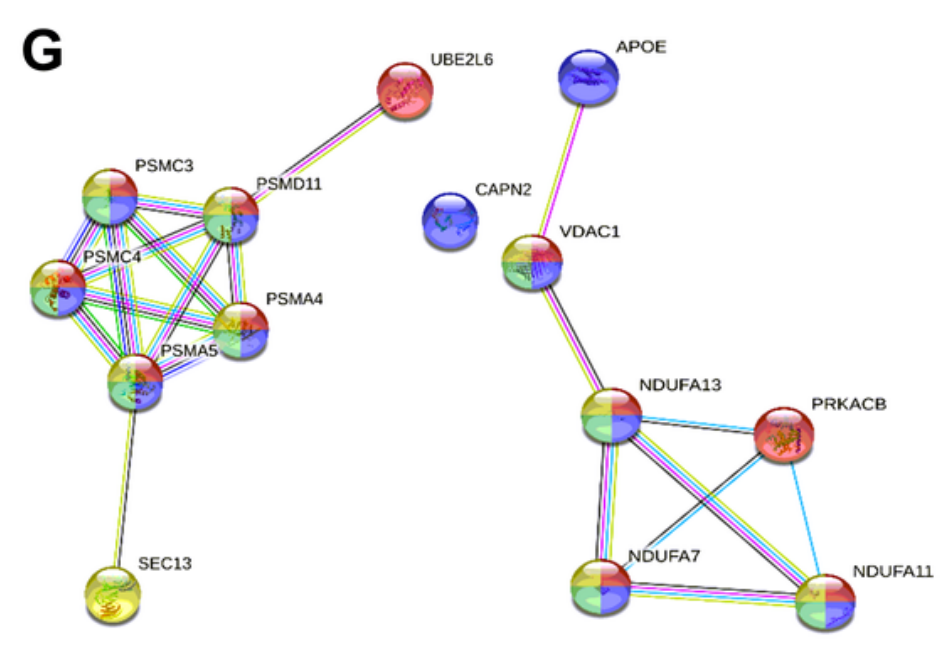

Figure 4

Bioinformatics analysis of the DEPs of plasma and PBMCs between children with ASD and healthy controls. (A) The key biological process (BP) associated with DEPs of plasma. Number of proteins 
associated with each category is shown on the right of each term bar. (B) PPI network linked to the plasma DEPs. The BP shown by the color of the bar chart in A is consistent with that shown by the same color in B. (C)-(E). The key KEGG pathways associated with DEPs of PBMCs. (F) The DEPs of PBMCs linked to the KEGG pathways of neurological diseases. Number of proteins associated with each category is shown on the right of each term bar. (G) PPI network linked to the DEPs of PBMCs associated with the KEGG pathways of neurological diseases. The pathways represented by the same color in $\mathrm{F}$ and $\mathrm{G}$ are consistent.
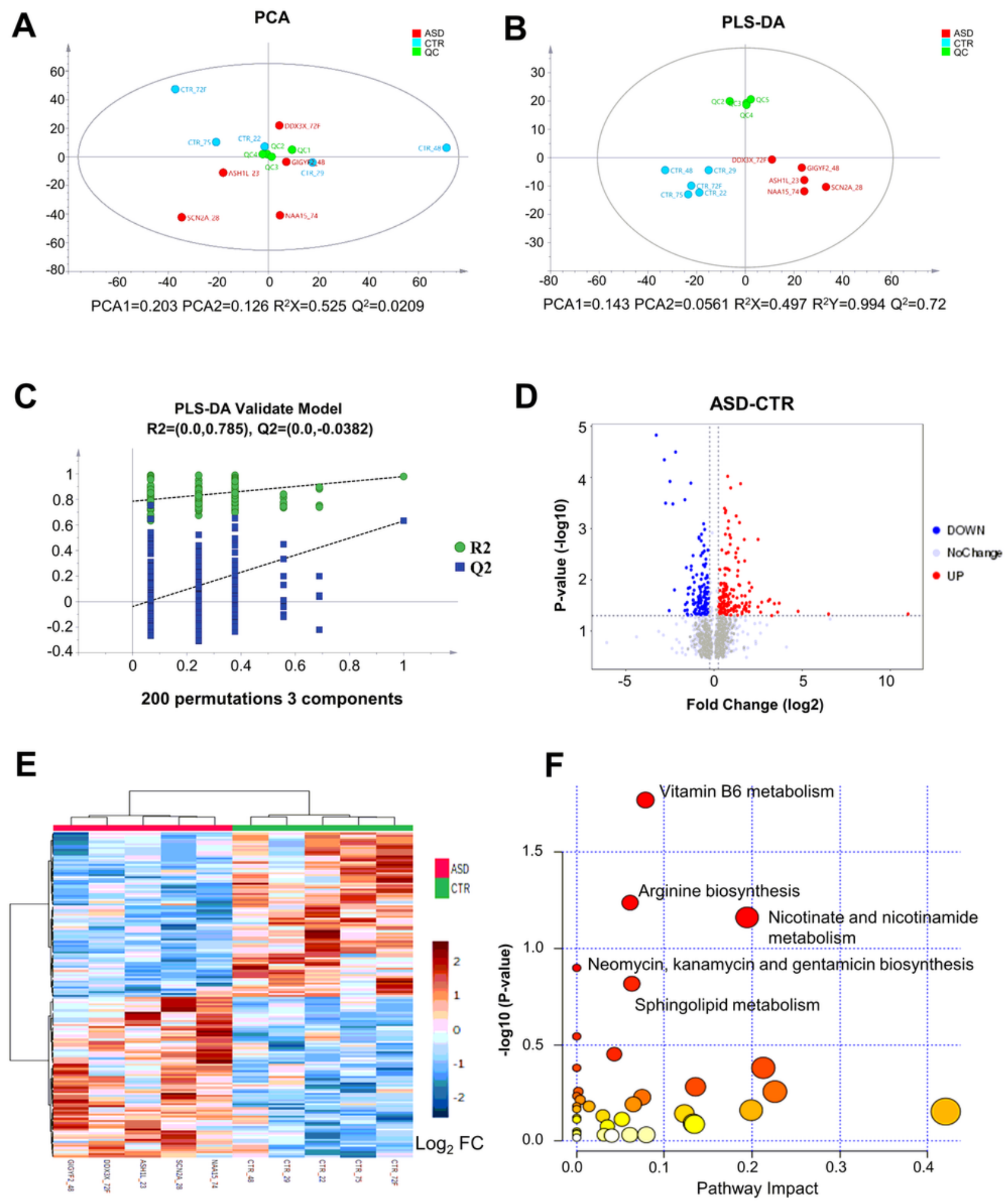


\section{Figure 5}

Statistical and bioinformatics analysis of metabolites detected by mass spectrometry in positive ion mode. (A) PCA analysis the metabolites of the case and the control group. (CTR). (B) PLS-DA analysis the metabolites of the cases and the control group. (C) PLS-DA model was verified by permutation test. (D) Volcano plot analysis of differential metabolites between children with ASD and healthy controls. The $\log 2$ fold change $(F C)$ is plotted versus the $-\log 10$ of the $p$ value. Blue and red dots: hits with $p<0.05$ and $|\log 2 \mathrm{FC}|>0.26$. (E) Cluster analysis the differential metabolites between children with ASD and healthy controls. (F) Metabolic pathways related to differential metabolites between children with ASD and healthy controls. 

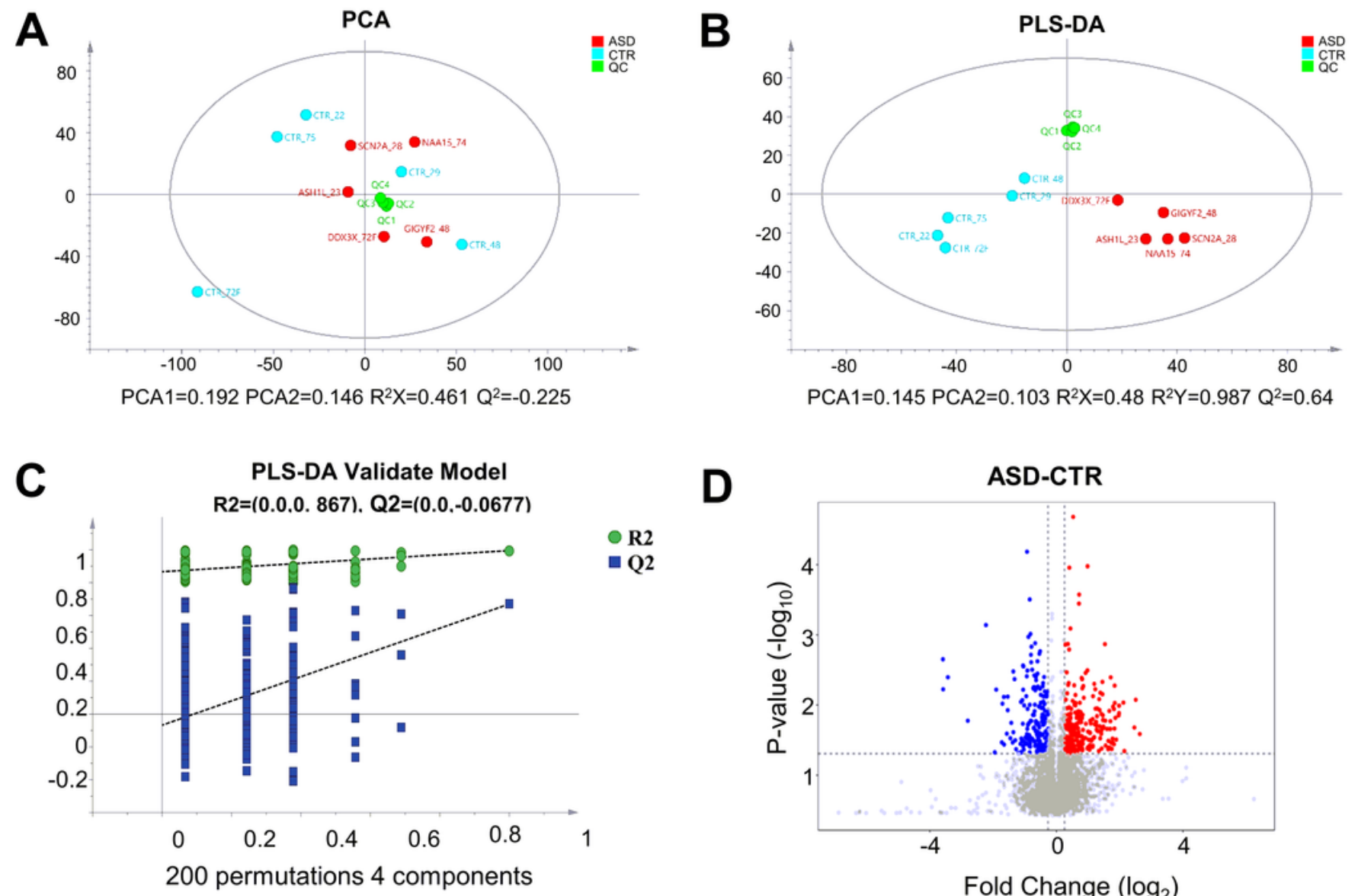

D
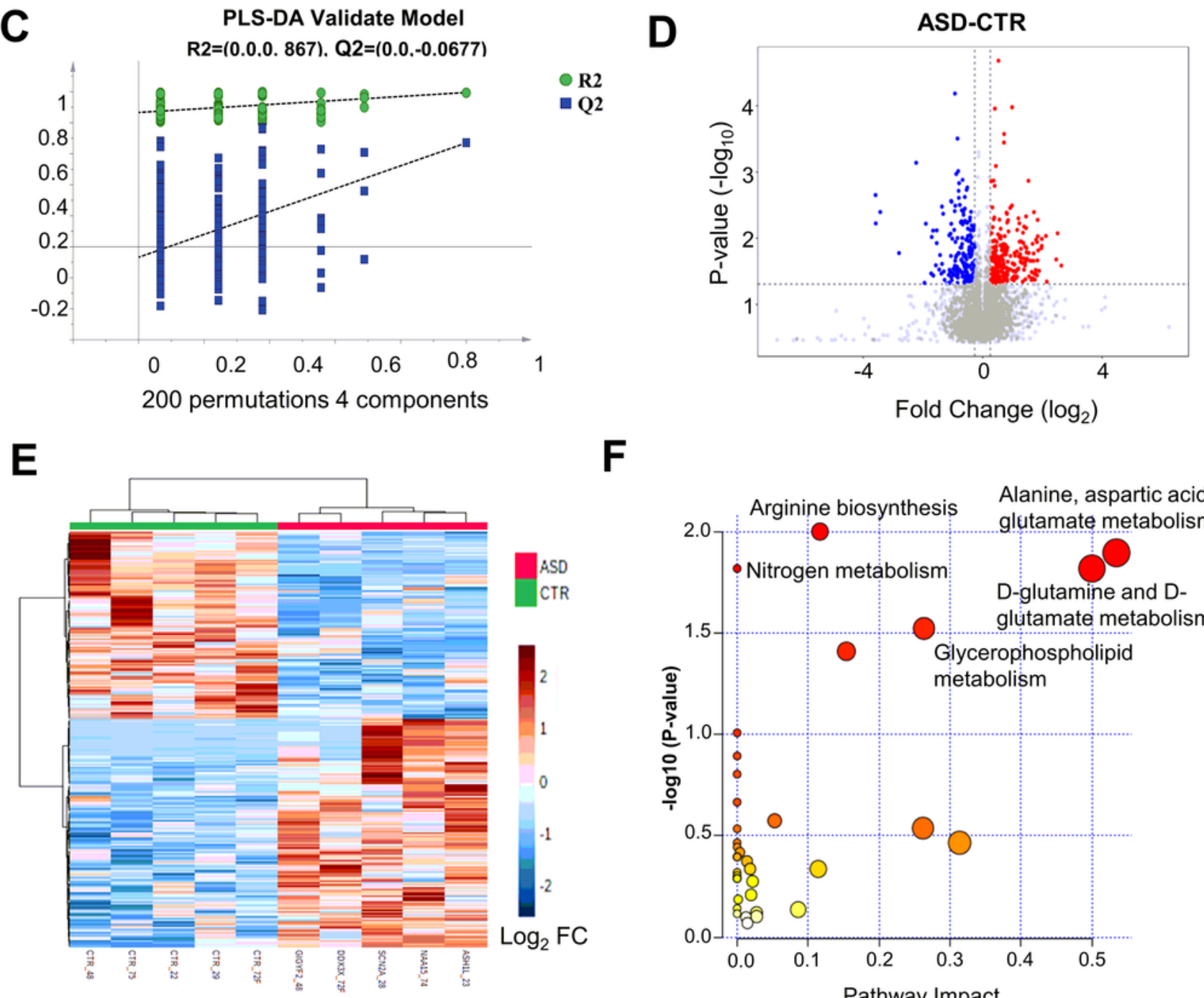

$\mathbf{F}$

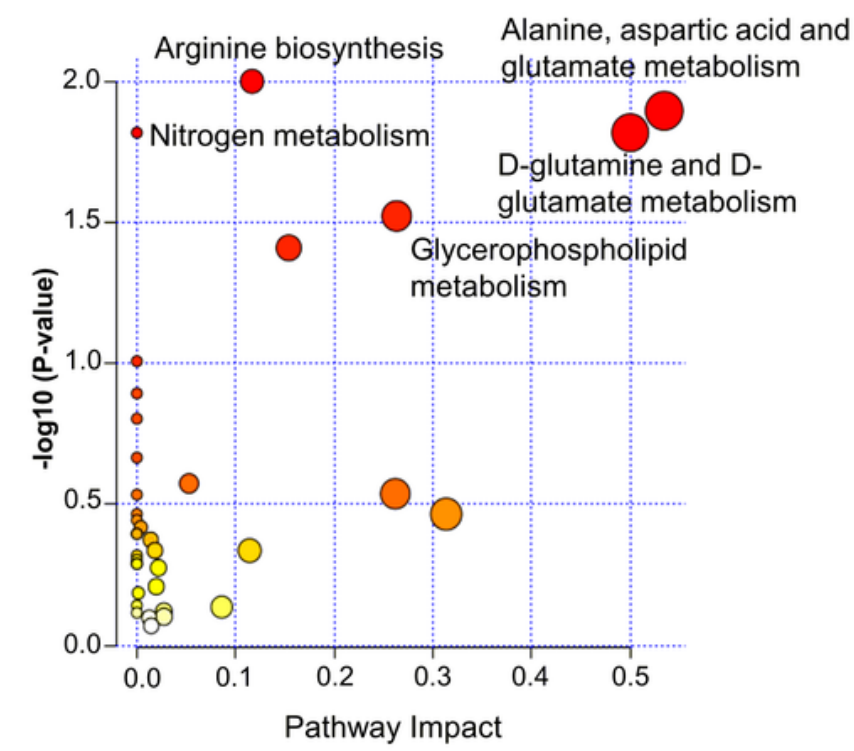

\section{Figure 6}

Statistical and bioinformatics analysis of metabolites detected by mass spectrometry in negative ion mode. (A) PCA analysis the metabolites of the case and the control group. (B) PLS-DA analysis the metabolites of the cases and the control group. (C) PLS-DA model was verified by permutation test. (D) Volcano plot analysis of differential metabolites between children with ASD and healthy controls. The $\log 2$ fold change $(F C)$ is plotted versus the - $\log 10$ of the $p$ value. Blue and red dots: hits with $p<0.05$ and 
$|\log 2 \mathrm{FC}|>0.26$. (E) Cluster analysis the differential metabolites between children with ASD and healthy controls. (F) Metabolic pathways related to differential metabolites between children with ASD and healthy controls.

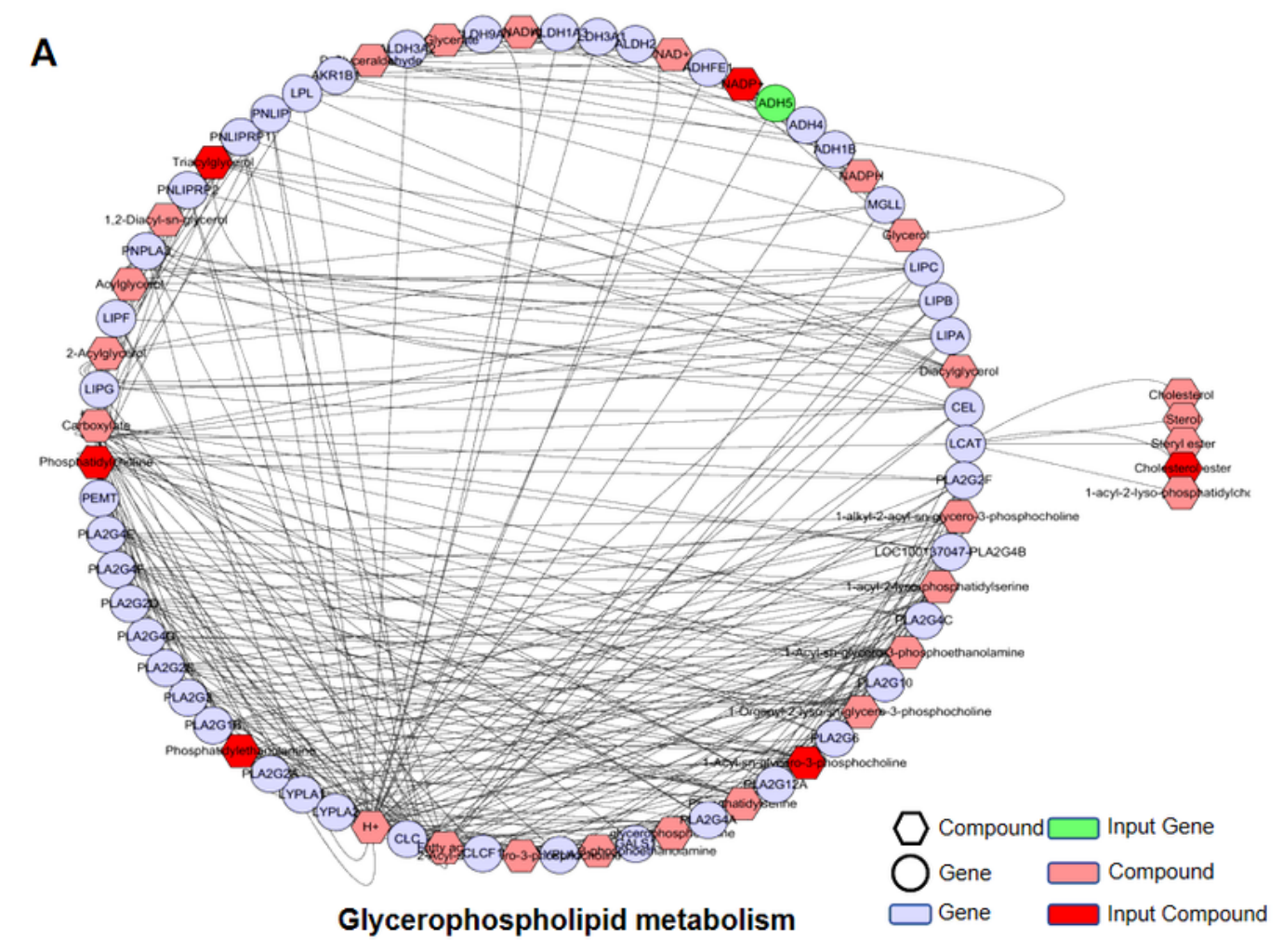

B

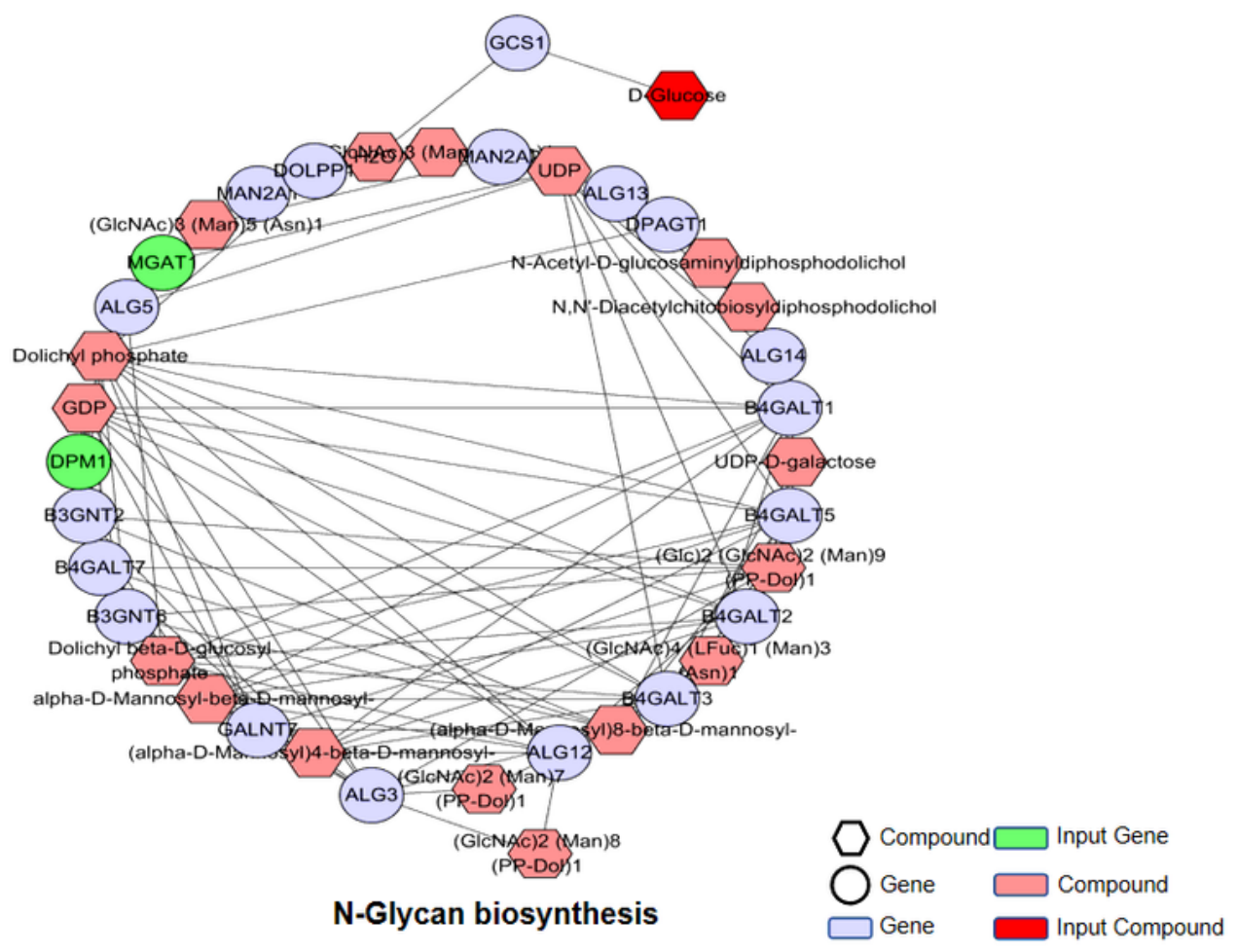

Figure 7

Integrated analysis of proteomics and metabolomics results. (A) Glycerophospholipid metabolism was associated with the DEPs of proteomics and the differential metabolites of metabolomics. (B) N-Glycan 
biosynthesis was associated with the DEPs of proteomics and the differential metabolites of metabolomics.

\section{Gene heterogeneity}

(ASH1L, SCN2A, GIGYF2,

NAA15, and DDX3X)

Plasma and PBMCs proteomics

- Complement and coagulation cascades

- Inflammatory and immune response

- Mitochondrial dysfunction

- Proteasome, ubiquitin mediated proteolysis, and unfolded protein response and ER stress

- Four risk genes of ASD are involved in DEPs of PBMCs (i.e., CCT4, CSDE1, DDX3X, and SLC9A9)

\section{Plasma metabolomics}

\section{- Amino acid}

alanine, aspartate and glutamate, arginine biosynthesis, and D-glutamine and D-glutamate

\section{- Vitamins}

nicotinate and nicotinamide, and vitamin B6

\section{- Lipids}

glycerophospholipid and sphingolipid

- Common changes in the profiling of proteomics and metabolomics

- Consistent or inconsistent changes in the brain and periphery, or different functions

- The relationship and integration of protein and metabolism

\section{Figure 8}

Common mechanisms and potential biomarkers can be obtained in children with ASD with different risk gene. Bold letters indicate that these pathways are related to the DEPs and differential metabolites in this study.

\section{Supplementary Files}

This is a list of supplementary files associated with this preprint. Click to download.

- Additionalfile1.docx

- Additionalfile2.docx

- Additionalfile3.docx

- Additionalfile4.docx 